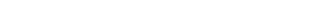

\title{
Traumatismo traqueal cervical por alambres o cuerdas tendidos sobre la vía pública. Una lesión sui géneris
}

\author{
Cervical tracheal trauma by wires or cirds stretched \\ over public way. A sui generis lesion
}

\author{
Edelberto Fuentes-Valdés* \\ *Hospital Clínico Quirúrgico «Hermanos Ameijeiras», Universidad de Ciencias Médicas de La Habana. La Habana, Cuba.
}

\begin{abstract}
RESUMEN. La tráquea cervical está en gran riesgo de lesiones por traumatismos del cuello. Objetivo: Presentar tres pacientes que sufrieron lesiones traqueales abiertas producidas por alambres (2/3) y contusas, con una cuerda (1/3), que se encontraban tendidos sobre la vía pública, mientras viajaban en motocicletas. Material y métodos: Estudio descriptivo sobre una base de datos prospectiva de las afecciones de la vía respiratoria. Los pacientes fueron atendidos, primariamente, en otros centros hospitalarios del país, para más tarde ser remitidos a nuestro nosocomio para el tratamiento de las secuelas. Se estudió el sexo y la edad, localización y tipo de la lesión, tratamiento definitivo, complicaciones y resultado final. Resultados: Todos los pacientes (sexo masculino) presentaban una traqueostomía al momento de la recepción como método para garantizar la ventilación. El primero (31 años) sufrió una lesión abierta. Le realizaron resección traqueal y varias sesiones de tratamiento endoscópico (láser) y lo remitieron por recidiva de la estenosis. De manera inicial, se colocó un tubo en T, por lesión subglótica compleja e inflamación significativa. En el posoperatorio presentó un granuloma que se trató con antibióticos y corticosteroides. Con la resección subglótica ulterior se obtuvo un resultado satisfactorio. El segundo enfermo (20 años) tuvo fractura contusa del cartílago cricoides y primeros anillos traqueales al chocar contra una cuerda. Se le realizó resección subglótica con resultados excelentes. El último (35 años) sufrió una lesión abierta (alambre). Presentaba parálisis recurrencial bilateral. Se practicó la cordoaritenoidectomía posterior y, finalmente, la resección subglótica. El resultado fue satisfactorio. Conclusiones: La tráquea cervical está expuesta a lesiones graves en casos de traumatismo cervical. El mecanismo de producción descrito es muy raro, en tal medida que no encontramos ningún caso en la literatura revisada. El tratamiento de las secuelas en los supervivientes podría tener resultados satisfactorios si son tratados en centros hospitalarios y mediante cirujanos con experiencia en la cirugía traqueal.
\end{abstract}

Palabras clave: Traumatismo cervical, tráquea, alambres, cuerdas, traumatismo traqueal.

Correspondencia:

\section{Dr. Edelberto Fuentes-Valdés}

Hospital Clínico Quirúrgico Hermanos Ameijeiras,

Universidad de Ciencias Médicas de La Habana.

Correo electrónico: efuentes@infomed.sld.cu

Recibido: 09-XI-2020; aceptado: 26-III-2021.

Citar como: Fuentes-Valdés E. Traumatismo traqueal cervical por alambres o cuerdas tendidos sobre la vía pública. Una lesión sui géneris. Neumol Cir Torax. 2021; 80 (1): 39-41. https://dx.doi.org/10.35366/99452
ABSTRACT. Cervical trachea is at high risk of lesions from cervical trauma. Objective: To present three patients who underwent open cervical tracheal lesions with wires (2/3) or closed lesions with a string (1/3), hanged out over public way, while travelling in a motorcycle. Material and methods: Descriptive study on a prospective data base related to airway diseases. All were treated primarily in other hospital and later referred to us to sequela treatment. Sex, age, location and type of lesion, definitive treatment, complications and final results were studied. Results: All three patients (males) were referred to us after initial treatment. First patient (31 years) underwent an open trauma. He was treated with a tracheal resection and endoscopic methods. After tracheal stenosis recurrence he was referred to our hospital. Firstly, we inserted a T tube, because of a complex subglottic lesion and important inflammation. A postoperative granuloma was treated conservatively. A satisfactory result was obtained by subglottic resection. Second patient (20 years) had a contused tracheal trauma due to striking against a corde. We performed a subglottic resection with excellent results. The last patient (35 years) underwent an open trauma (with a wire). He had a bilateral recurrent nerve paralysis. In the first time posterior cordoaritenoidectomy was done, and posteriorly a subglottic resection. Final result was satisfactory. Conclusions: Cervical trachea is exposed to severe lesions in cases of cervical trauma. Described production mechanism is so rare that were not found cases in the reviewed literature. Treatment of sequela in survivors could have satisfactory results if treated in centers and surgeons with experience in tracheal surgery.

Keywords: Cervical trauma, trachea, wires, cords, tracheal trauma.

\section{INTRODUCCIÓN}

El traumatismo de la vía respiratoria (VR) suele ser una lesión grave que amenaza la vida. Es el resultado de lesiones contundentes o penetrantes en el cuello y el tórax, además de lesiones iatrógenas, principalmente durante la práctica de una broncoscopia rígida o una intubación traqueal. ${ }^{1} \mathrm{El}$ diagnóstico rápido es obligatorio para la supervivencia del enfermo. ${ }^{2}$ El tratamiento suele ser difícil e incluye, en primer 
lugar, obtener una VR segura y permeable que permita la ventilación adecuada y, a continuación, la reparación de la lesión con el menor impacto posible sobre la función respiratoria y la calidad de vida de los enfermos. ${ }^{3}$

Los pacientes con ruptura completa de la tráquea, causada por una lesión penetrante en el cuello, pueden ser salvados mediante la intubación, con carácter urgente, a través de la herida abierta. ${ }^{4}$ Desde el punto de vista etiológico, el traumatismo traqueal se clasifica en iatrógeno y traumático, y la localización en cervical y torácica. A su vez, las traumáticas se dividen en abiertas y contusas. Las abiertas consisten en heridas por armas de fuego o por objetos agudos.

El objetivo principal de este artículo es la revisión de un tipo de traumatismo traqueal cervical sui géneris, debido al mecanismo de producción: choque contra alambres (2) o cuerda (1) que se encontraban tendidos sobre la vía pública, mientras se conducían motocicletas.

\section{MATERIAL Y MÉTODOS}

Se realizó un estudio descriptivo sobre una base de datos prospectiva (Access 2010) de afecciones quirúrgicas de la tráquea. Los pacientes, del sexo masculino, con edades de 20, 31 y 35 años, fueron tratados entre 1998 y 2001. La lesión traqueal se produjo mientras viajaban en motocicletas e impactaron contra un alambre (2/3) o una cuerda (1/3) que se encontraban tendidos sobre la vía pública. Todos fueron tratados, inicialmente, en otros centros hospitalarios, donde lograron el control de la VR. Después fueron remitidos para el tratamiento de las secuelas. Los dos pacientes que sufrieron el traumatismo con un alambre presentaron lesiones abiertas, mientras que el que se proyectó contra una cuerda presentó una lesión contusa con fractura del cartílago cricoides (Figura 1). El tratamiento inicial en los centros de origen consistió en traqueostomía y resección traqueal para los tres pacientes y varias sesiones de tratamiento endoscópico (láser) para uno de ellos. Con el fracaso de la resección fueron remitidos a nuestro centro para el tratamiento definitivo. El resultado se consideró satisfactorio cuando el paciente podía reintegrarse a una vida normal, pero tenía una voz débil, y excelente si el paciente pudo reincorporarse a su vida normal, sin limitaciones y la voz era normal.

\section{RESULTADOS}

Al paciente con resección traqueal y tratamiento endoscópico previo se le colocó un tubo en T inicialmente, por inflamación local significativa. En los tres se encontró estenosis subglótica, aunque fueron remitidos con diagnóstico de estenosis traqueal. El enfermo con traumatismo traqueal contuso tenía una fractura del cartílago cricoides (Figura 1).
En todos se practicó resección laringotraqueal subglótica por la técnica de Pearson (Figura 2). Un paciente tenía parálisis recurrencial bilateral, por lo que en primera instancia se le realizó una cordoaritenoidectomía posterior y después la resección laringotraqueal. Sólo hubo una complicación, granuloma de la línea de sutura en el paciente previamente operado. Con un seguimiento de 18, 24 y 62 meses, dos enfermos tuvieron resultados satisfactorios, debido a debilidad de la voz, y uno excelente.

\section{DISCUSIÓN}

La tráquea cervical está en gran riesgo de lesiones por traumatismos del cuello. Como quiera que los pacientes hayan sido tratados inicialmente en otros centros hospitalarios, la discusión se centró en el tratamiento definitivo de las secuelas del traumatismo traqueal. La lesión de nuestros pacientes se produjo cuando el motociclista encontró en su camino alambres o cuerdas tendidos sobre la vía pública, generalmente durante actividades festivas. En la revisión bibliográfica no se encontraron reportes de casos con el mecanismo de lesión motivo del presente artículo.

Las lesiones penetrantes de la región cervical se presentan entre $5-10 \%$ de todos los casos de traumatismos que ingresan en un área de urgencias. ${ }^{5}$ La incidencia verdadera todavía es desconocida, porque de 30 a 80\% de las víctimas fallecen en la escena del accidente. ${ }^{6}$

En pacientes como los ya presentados, nunca se insistirá bastante en los cuidados intraoperatorios, cuando se realiza la movilización circunferencial de los bordes lesionados, porque aumenta el riesgo de la desvascularización de los extremos a anastomosarse y la posibilidad de dehiscencia

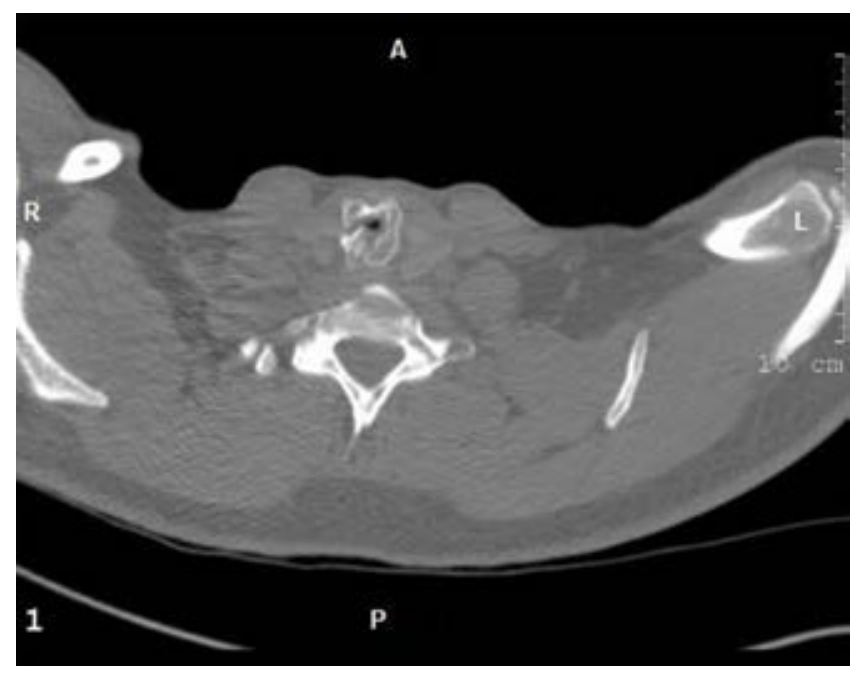

Figura 1: Tomografía axial computarizada. Vista axial. Se observa la fractura del cartílago cricoides y el pequeño orificio de la luz de la VR a ese nivel. 

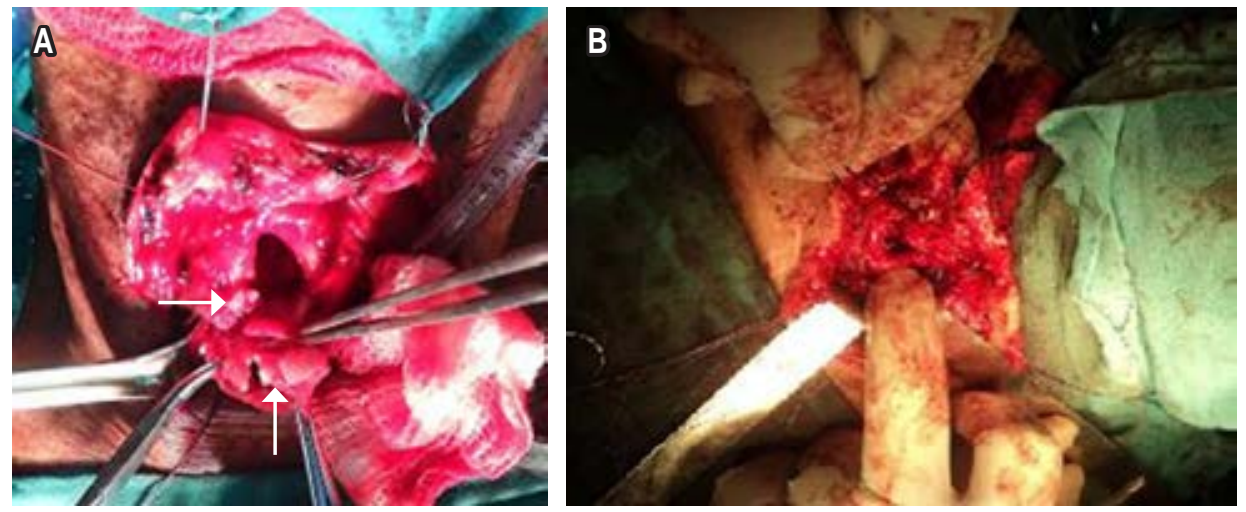

Figura 2:

Resección subglótica. A) Se observa la resección del plato anterior y lateral del cricoides fracturado (flechas). B) Anastomosis de la tráquea proximal con la laringe (cartílago tiroides en paredes laterales y anterior, y la pared membranosa traqueal con el plato posterior del cricoides).

de la anastomosis, estenosis tardía o infección. ${ }^{3}$ El autor usa suturas absorbibles a largo plazo como Vicryl, PDS o ácido poliglicólico, monofilamento calibre 4/0 para la pared membranosa y $3 / 0$ para la cartilaginosa.

A pesar de la complejidad de la intervención, sólo un paciente presentó una complicación: granuloma en la línea de sutura que se resolvió con tratamiento conservador a base de antibióticos y corticosteroides orales. En general, las complicaciones se pueden ver hasta en $25.8 \%$ de los pacientes con traumatismo, las graves se asocian con infecciones secundarias a larga estadía en unidades de cuidados intensivos y dehiscencia anastomótica. ${ }^{7}$ Las complicaciones a largo plazo incluyen estenosis ${ }^{8}$ y trastornos de la fonación por lesiones de la laringe y de los nervios laríngeos recurrentes. ${ }^{9}$ Es probable que los resultados obtenidos se deban, en cierta medida, a la experiencia de varios años en la cirugía traqueal del autor.

\section{CONCLUSIONES}

En resumen, la tráquea cervical está en gran riesgo de lesiones por traumatismos del cuello. El artículo actual hace referencia a una causa de lesión sui géneris por el mecanismo de producción ya expuesto. El control inicial de la VR es fundamental para salvar la vida del lesionado. Aunque complejo, el tratamiento definitivo puede alcanzar resultados satisfactorios y excelentes, siempre y cuando el paciente sea tratado en centros hospitalarios y mediante especialistas con experiencia en la cirugía traqueal.

\section{REFERENCIAS}

1. Prokakis Ch, Koletsis EN, Dedeilias P, Fligou F, Filos K, Dougenis D. Airway trauma: a review on epidemiology, mechanisms of injury, diagnosis and treatment. J Cardiothoracic Surg. 2014:9:117. https:// doi.org/10.1186/1749-8090-9-117

2. Vázquez-Minero JC, Morales-Gómez J, Guzmán-de Alba E, ÁlvarezÁlvarez S, Trueba-Lozano D, Cervantes-Silva Y, et al. Trauma traqueal penetrante. Recomendaciones para su manejo. A propósito de un caso. Neumol Cir Torax. 2018;77(4):286-292.

3. Dougenis D. Trauma of the tracheobronchial tree. Arch Hellen Med. 2002;19(3):258-269.

4. Zhao Z, Zhang T, Yin X, Zhao J, Li X, Zhou Y. Update of the diagnosis and treatment of tracheal and bronchial injury. $J$ Thorac Dis. 2017;9(1):E50-E56. https://doi.org/10.21037/jtd.2017.01.19

5. Palacios-Zertuche JT, Montero-Cantú CA, Guerrero-Hernández AJ, Salinas-Domínguez R, Pérez-Rodríguez E, Muñoz-Maldonado GE. Herida por proyectil de arma de fuego en cuello con lesión traqueoesofágica y colocación de parche muscular de esternotiroideo. Neumol Cir Torax. 2016;75(3):222-226.

6. Johnson SB. Tracheobronchial injury. Semin Thorac Cardiovasc Surg. 2008;20(1):52-57. https://doi.org/10.1053/j.semtcvs.2007.09.001

7. Abernathy JHIII, Reeves ST. Airway catastrophes. Curr Opin Anaesthesiol. 2010;23(1):41-46. https://doi.org/10.1097/aco.0b013e328333b768

8. Carretta A, Melloni G, Bandiera A, Negri G, Voci C, Zannini P. Conservative and surgical treatment of acute posttraumatic tracheobronchial injuries. World J Surg. 2011;35(11):2568-2574. https://doi.org/10.1007/s00268-011-1227-z

9. Welter S, Cheufou D, Darwiche K, Stamatis G. Tracheal injuries, fistulae from bronchial stump and bronchial anastomoses and recurrent laryngeal nerve paralysis: management of complications in thoracic surgery. Chirurg. 2015;86(5):410-418. https://doi.org/10.1007/s00104-014-2862-3

Conflicto de intereses: El autor declara no tener conflicto de intereses. 